\title{
Real-expansive flows and topological dimension
}

\author{
H. B. KEYNES $\dagger$ AND M. SEARS \\ From the School of Mathematics, University of Minnesota, USA; and the Department \\ of Applied Mathematics, University of the Witwatersrand, Johannesburg, South Africa
}

(Received 18 February 1981)

\begin{abstract}
We examine generalizations of $\mathrm{R}$. Mañé's results on the topological dimension of spaces supporting an expansive homeomorphism to the case of real-expansive flows. We show that a space supporting a real-expansive flow must be finite dimensional, and a minimal real-expansive flow not exhibiting a type of spiral behaviour must be one-dimensional. This latter class includes all known examples and a slight generalization of Axiom A flows. These results are obtained by introducing a new concept of stable and unstable sets for real flows, and examining real-expansive flows in terms of these sets.
\end{abstract}

\section{Introduction}

In [5] R. Mañé showed that minimal sets of expansive homeomorphisms are zero dimensional thus proving that every minimal expansive homeomorphism is a subshift. In [2] R. Bowen obtained the analogous result for minimal sets of Axiom A flows. In this paper we introduce a new concept of stable and unstable sets for real flows which enables us (using Mañé's approach) to obtain Bowen's result without using the machinery of Markov partitions, and to generalize it to certain other real expansive flows. Mañé also proved that every space supporting an expansive homeomorphism must have finite topological dimension and we obtain the corresponding result for real-expansive flows.

Throughout the paper $(X, \mathbb{R})$ represents a real flow without fixed points on a compact metric space $X$. $d$ will denote a metric on $X$ and the action of $t \in \mathbb{R}$ on $x \in X$ is written $x t$. We shall assume that $(X, \mathbb{R})$ is not trivial in the sense that it consists merely of a finite number of orbits.

\section{Stable and unstable sets for real flows}

The obvious extension of the $\varepsilon$-stable sets used by Mañé for homeomorphisms in [5] and by Bowen for Axiom A flows in [2] to general real flows would be (at $x$ ) $\{y ; d(x t, y t)<\varepsilon$ for all $t \geq 0\}$. However, the example given below shows that there are flows such that, for any $x$, this set consists only of points of the form $x t$ for $t$ sufficiently small (such points must always belong to the set) and there are no asymptotic orbits. The definition of real expansiveness indicates that we could not 
expect this to be a useful idea of $\varepsilon$-stable set in any case. These considerations motivate the introduction of a concept of stable and unstable sets which allows time delays.

(2.1) Definition. A real flow $(X, \mathbb{R})$ is called real expansive [3] if it satisfies the following condition.

For every $\varepsilon>0$ there is a $\delta>0$ such that, if $s$ is any continuous function $(s: \mathbb{R} \rightarrow \mathbb{R})$ satisfying $s(0)=0$, then for each $x \in X$ and $y \in X$ with $y \notin x(-\varepsilon, \varepsilon)$ there is $t \in \mathbb{R}$ with $d(x t, y s(t))>\delta$.

(2.2) Example. We construct a minimal expansive real flow which has no positively or negatively asymptotic orbits and which is not a finite number of orbits. Note that this is very far removed from the case of Axiom A flows (see proposition 1.3 of [1]). The example is a suspension of a Sturmian minimal set and so is expansive by theorem 6 of [3].

Let $\alpha<\frac{1}{2}$ be irrational and $\tau$ the $\operatorname{map} \tau(x)=x+\alpha(\bmod 1)$ from $[0,1)$ onto $[0,1)$. Create a Sturmian set by 'splitting' along the orbit of 0 under $\tau$. This gives a minimal expansive flow $(M, \phi)$, say. Now let $\left\{x_{n}\right\}=\{n \alpha\}$ and choose an increasing sequence of positive integers $\left\{n_{j}^{+}\right\}$and a decreasing sequence of negative integers $\left\{n_{j}^{-}\right\}$such that $x_{n_{j}^{+}}$is strictly decreasing to 0 and $x_{n_{j}^{-}}$is strictly increasing to 1 . Next find a sequence $\left\{\delta_{j}\right\}$ decreasing to 0 such that, defining $I_{j}^{+}=\left[x_{n_{i}}^{+}, x_{n_{j}}^{+}+\delta_{j}\right]$ and $I_{j}^{-}=$ $\left[x_{n_{j}}^{-}-\delta_{j}, x_{n_{j}}^{-}\right], I_{j}^{+} \cap I_{k}^{+}=\varnothing(j \neq k), I_{j}^{-} \cap I_{k}^{-}=\varnothing(j \neq k)$ and $I_{j}^{+} \cap I_{k}^{-}=\varnothing$ for all $j$ and $k$. Define a function $f_{j}$ on $I_{j}^{+} \cup I_{j}^{-}$by $f_{j}\left(x_{n_{j}}^{ \pm}\right)=1+(1 / j), f_{j}\left(x_{n_{j}}^{ \pm} \pm \delta_{j}\right)=1$ and by linearity between the endpoints of each of the two intervals. Now define a function on $[0,1)$ by $f(x)=f_{j}(x), x \in I_{j}^{+} \cup I_{j}^{-}$and $f(x)=1$ otherwise. Note that since the discontinuities of $f$ occur at the points $\left\{x_{n_{i}}^{ \pm}\right\}, f$ can be extended to a continuous function on $M$.

Now form the suspension flow $\left(M_{f}, \mathbb{R}\right)$ of $(M, \phi)$ under $f$. Note that, if two points are real asymptotic in this flow, then (eventually) their base points are asymptotic under $\phi$ in $M$. However, the only such points are of the form $\phi^{k} 0^{-}$and $\phi^{k} 0^{+}$and so the only candidates for real asymptotic points are of the form $\left(\phi^{N} 0^{-}, u\right)$ and $\left(\phi^{N} 0^{+}, v\right)$ and, without loss of generality, we may assume $u<1$ and $v<1$. Now if $n_{i}^{+}<N \leq n_{i+1}^{+}$, then for each $j>1$ we can find $t_{j} \geq 0$ such that

$$
\left(\phi^{N} 0^{+}, v\right) t_{j}=\left(\phi^{n_{i+j}^{+}} 0^{+}, v\right) \text { but }\left(\phi^{N} 0^{-}, u\right) t_{j}=\left(\phi^{n_{i+i}^{+}} 0^{-}, u+\sum_{k=i+1}^{i+j-1} \frac{1}{k}\right)
$$

and so clearly these points are not positively asymptotic. Reversing the roles of $0^{-}$ and $\mathrm{O}^{+}$in this argument shows that they are not negatively asymptotic either.

All our analysis for a flow will be carried out relative to a fixed collection of cross-sections with special properties. This enables us to use the local product nature of the flow.

(2.3) Definition. $S \subset X$ is called a local cross-section of time $\zeta>0$ for the flow $(X, \mathbb{R})$ if $S$ is closed and $S \cap x[-\zeta, \zeta]=\{x\}$ fo, all $x \in S$.

If $S$ is a cross-section of time $\zeta$, the action maps $S \times[-\zeta, \zeta]$ homeomorphically onto $S[-\zeta, \zeta]$. By the interior $S^{*}$ of $S$ we mean the set $S \cap$ int $(S[-\zeta, \zeta])$. Note that $S^{*}(-\varepsilon, \varepsilon)$ is open in $X$ for any $0<\varepsilon<\zeta$. 
A theorem of Whitney ([6], p. 270) asserts that, for each $x \in X$, there is a local cross-section $S_{x}$ of time $\nu_{x}$ with $x \in S_{x}^{*}$. The following lemma is obtained by carefully modifying the proof of lemma 7 of [3].

(2.4) LEMMA. There is $a \zeta>0$ such that for each $\alpha>0$ we can find a finite family $\mathscr{S}=\left\{S_{1}, S_{2}, \ldots, S_{k}\right\}$ of pairwise disjoint local sections of time $\zeta$ and diameter at most $\alpha$ and a family $\mathscr{T}=\left\{T_{1}, T_{2}, \ldots, T_{k}\right\}$ with $T_{i} \subset S_{i}^{*}(i=1,2, \ldots, k)$ such that

$$
X=T^{+}[0, \alpha]=T^{+}[-\alpha, 0]=S^{+}[0, \alpha]=S^{+}[-\alpha, 0]
$$

where $T^{+}=\bigcup_{i=1}^{k} T_{i}$ and $S^{+}=\bigcup_{i=1}^{k} S_{i}$

We can now define stable and unstable sets allowing time delays in terms of a fixed collection of sections. Choose $\zeta$ as in lemma 2.4 and $\alpha$ such that $3 \alpha<\zeta$ and select appropriate collections of sections $\mathscr{S}$ and $\mathscr{T}$ as in that lemma. For convenience, if $S \in \mathscr{S}$ then we shall denote by $T$ the appropriate element of $\mathscr{T}$ such that $T \subset S^{*}$.

Now let $\beta$ be the minimum time between sections of $\mathscr{S}$ i.e.

$$
\beta=\sup \left\{\delta>0 ; \text { for any } x \in S^{+} \text {we have } x(0, \delta) \cap S^{+}=\varnothing\right\} \text {. }
$$

Note that $0<\beta \leq \alpha$. Let $\rho>0$ satisfy $5 \rho<\zeta$ and $2 \rho<\beta$.

We next define a first return map $\phi$ on $T^{+}$. If $x \in T^{+}$then $\phi(x)=x t$, where $t$ is the smallest positive time such that $x t \in T^{+}$. Note that $\beta \leq t \leq \alpha$. Also for each $S_{i} \in \mathscr{S}$, let $D_{\rho}^{i}=S_{i}[-\rho, \rho]$ and define a projection map $P_{\rho}^{i}: D_{\rho}^{i} \rightarrow S_{i}$ by $P_{\rho}^{i}(x)=x t$, where $x t \in S_{i}$ and $|t| \leq \rho$. Since $2 \rho<\zeta, P_{\rho}^{i}$ is well defined, continuous and onto $S_{i}$. (If we do not need to specify the set in $\mathscr{S}$, we shall write $D_{\rho}^{i}$ as $D_{\rho}$ and $P_{\rho}^{i}$ as $P_{\rho}$.) Also choose $0<\varepsilon<\frac{1}{2} \beta$ such that, if $x, y \in S, d(x, y) \leq \varepsilon$ and $t$ is such that $|t| \leq 3 \alpha$ and $x t \in T$, then $y t \in D_{\rho}$.

Using this notation, we can set up a 'shadowing' orbit of $y$ relative to the $\phi$ orbit of any point $x \in T^{+}$. For, if $y$ is sufficiently close to $x$, the orbit of $y$ will cross $S$ at a time near the time when the orbit of $x$ crosses $T$. We can now repeat this idea using $\phi(x)$ and the point where the $y$ orbit crosses $S$ as our base points and continue in this way as long as these pairs of points remain close enough. Formally, if $x \in T$ and $y \in S$ with $d(x, y) \leq \varepsilon$, we can define a set of points $\left\{y_{0}, y_{1}, \ldots, y_{n}\right\}$ on the orbit of $y$ by $y_{0}=y$, and $y_{i}=P_{\rho}\left(y_{i-1} t\right)$, where $t$ is the smallest positive time such that $\phi^{i}(x)=\phi^{i-1}(x) t$, and we can continue this construction as long as $d\left(\phi^{i} x, y_{i}\right) \leq \varepsilon$. We thus obtain a time delayed $y$ shadow orbit along a piece of the orbit of $x$. Clearly we can proceed in the same way for negative powers of $\phi$. If there is possible confusion as to which point is being shadowed, we write $y_{i}^{x}$.

(2.5) Definition. Let $x \in T$ and $\eta<\varepsilon$. The $\eta$-stable set of $x$ is

$$
W_{\eta}^{s}(x)=\left\{y \in S ; d\left(\phi^{i} x, y_{i}\right)<\eta \quad \text { for all } i \geq 0\right\}
$$

and the $\eta$-unstable set of $x$ is

$$
W_{\eta}^{u}(x)=\left\{y \in S ; d\left(\phi^{i} x, y_{i}\right)<\eta \quad \text { for all } i \leq 0\right\} .
$$

This definition is certainly appropriate for the case when $\left(X_{f}, \mathbb{R}\right)$ is a suspension over an integer flow $(X, \psi)$. For clearly given $\eta^{\prime}>0$ there is an $\eta>0$ such that, if $y$ satisfies $d\left(\psi^{i} y, \psi^{i} x\right)<\eta$ for all $i \geq 0$, then $y s \in W_{\eta^{\prime}}^{s}(x t)$ where $x t \in T$ 
and $y s=P_{\rho}(y t)$. Thus points with stable orbits in the base produce points with stable orbits (in the sense of 2.5 ) in the suspension. It is equally straightforward to check the converse.

By the definition of $\varepsilon$, it is sufficient to consider subsets of the orbit and shadow orbit, $\left\{\phi^{l_{i}} x\right\}$ and $\left\{y_{l_{i}}\right\}$, say, with $\left\{l_{i}\right\}$ increasing and such that $\phi^{l_{i+1}} x=\left(\phi^{l_{i}} x\right) t$ for some $|t|<3 \alpha$. We formalize this idea as follows.

(2.6) LemMA. (i) Suppose $d\left(\phi^{i} x, y_{i}\right)<\varepsilon$ and $\phi^{i} x=\left(\phi^{i} x\right) t$ for some $|t| \leq 3 \alpha$, then $P_{\rho}\left(y_{i} t\right)=y_{j}$.

(ii) Suppose $d\left(\phi^{i} x, y_{i}\right)<\varepsilon$ and $y_{j}=y_{i}$ for some $|t| \leq 2 \alpha$, then $\left(\phi^{i} x\right) t \in D_{\rho}$ and $P_{p}\left(\left(\phi^{i} x\right) t\right)=\phi^{i} x$.

(iii) Given $\eta<\varepsilon$ there is a $\delta \leq \varepsilon$ such that if $x \in T^{+}$and $\left\{l_{i}\right\}$ is any increasing sequence of non-negative integers with $l_{0}=0$ such that for each $i, \phi^{l_{i+1}} x=\left(\phi^{l_{i}} x\right) t_{i}$ where $0<t_{i} \leq \alpha$, then if $y \in S^{+}$with $d\left(\phi^{l_{k}} x, y_{l_{k}}\right)<\delta$ for every $k, y \in W_{\eta}^{s}(x)$. A similar statement holds for $W_{\eta}^{u}(x)$.

Proof. (i) follows by the definition of $\varepsilon$ and a straightforward induction argument similar to that in the proof of (ii).

(ii) Without loss of generality, assume $j>i$. The claim holds for $j=i+1$, so assume it holds for $j=i+n$ for some $n \geq 1$. Suppose $y_{i+n}=y_{i} t$ and $y_{i+n+1}=y_{i+n} s$, where $0<t+s<2 \alpha$. Then

$$
\begin{gathered}
\phi^{i+n}(x)=\phi^{i}(x)(t+\eta), \quad \text { where }|\eta|<\rho \quad \text { and clearly } \\
\phi^{i+n+1}(x)=\phi^{i+n}(x)\left(s+\eta^{\prime}\right), \quad \text { where }\left|\eta^{\prime}\right|<\rho .
\end{gathered}
$$

Now

$$
\phi^{i+n+1}(x)=\phi^{i}(x)\left(t+s+\eta+\eta^{\prime}\right)
$$

and the result follows by (i) since $2 \rho<\alpha$ and $3 \rho<\zeta$.

(iii) Suppose $k>0$ is an integer between $l_{i}$ and $l_{i+1}$ and $\phi^{k}(x)=\phi^{l_{i}}(x) t$, where $0<t \leq \alpha$. By (i) we have $y_{k}=P_{\rho}\left(y_{l_{i}} t\right)$. Now by flow continuity and the continuity of $P_{\rho}$, we can find $\delta>0$ such that, if $x \in T$ and $y \in S$ and $d(x, y)<\delta$, then $d\left(x t, P_{\rho}(y t)\right)<$ $\eta$, where $x t \in T^{+}$and $0<t \leq \alpha$.

(2.7) THEOREM. $(X, \mathbb{R})$ is real expansive if and only if given collections of sections $\mathscr{S}$ and $\mathscr{T}$ and $\rho>0$, there is an $\eta>0$ such that for any $x \in T^{+}, W_{\eta}^{s}(x) \cap W_{\eta}^{u}(x)=\{x\}$.

Proof. First suppose $(X, \mathbb{R})$ is real expansive. Choose $0<\varepsilon_{0}<\zeta$ and $\varepsilon_{1}>0$ an expansive constant corresponding to $\varepsilon_{0}$. Let $\varepsilon>\eta>0$ such that, if $x \in T, y \in S$ and $d(x, y)<\eta$, then $d(x t, y s)<\varepsilon_{1}$ where, if $\phi x=x t_{1}$ and $y_{1}^{x}=y s_{1}$, then $0 \leq t \leq t_{1}, 0 \leq s \leq$ $s_{1}$ and $|s-t| \leq\left|s_{1}-t_{1}\right|$. Suppose $y \neq x, y \in W_{\eta}^{s}(x) \cap W_{\eta}^{u}(x)$ and $\left\{t_{n}\right\}$ and $\left\{s_{n}\right\}$ are the increasing bisequences such that $x t_{n}=\phi^{n} x$ and $y s_{n}=y_{n}$. Define a piecewise linear function $f$ from $\mathbb{R}$ onto $\mathbb{R}$ such that $f\left(t_{n}\right)=s_{n}$. Now for any $t \in \mathbb{R}, t=t_{n}+\sigma$ and $f(t)=s_{n}+\sigma^{\prime}$, where $0 \leq \sigma \leq t_{n+1}-t_{n}, 0 \leq \sigma^{\prime} \leq s_{n+1}-s_{n}$, and

$$
\left|\sigma-\sigma^{\prime}\right| \leq\left|\left(t_{n+1}-t_{n}\right)-\left(s_{n+1}-s_{n}\right)\right|
$$

for some $n$, so that

$$
d(x t, y f(t))=d\left(\left(\phi^{n} x\right) \sigma, y_{n} \sigma^{\prime}\right)<\varepsilon_{1}
$$

by the choice of $\eta$. But this contradicts expansiveness, since $y \notin x\left(-\varepsilon_{0}, \varepsilon_{0}\right)$ as $\varepsilon_{0}<\zeta$. 
Conversely, suppose that we are given collections of sections $\mathscr{S}$ and $\mathscr{T}$ and $\rho>0$ as in definition 2.5 and that for some $\eta>0$ and every $x \in T^{+}$we have $W_{\eta}^{s}(x) \cap W_{\eta}^{u}(x)=\{x\}$. Let $f$ be a continuous function from $\mathbb{R}$ to $\mathbb{R}$ satisfying $f(0)=0$. Let $x \in T, y \in S$ and $\left\{t_{n}\right\}$ and $\left\{s_{n}\right\}$ be such that $x t_{n}=\phi^{n} x$ and $y s_{n}=y_{n}$. We shall show that if $\left|f\left(t_{i}\right)-s_{i}\right|$ is sufficiently small for all $i$ then section separation forces $d(x t, y f(t))$ to be large for some $t$, while if this is not the case then the distance between $f\left(t_{i}\right)$ and $s_{i}$ forces $d(x t, y f(t))$ to become large for some $t$.

Choose $0<\delta<\zeta-\alpha-\rho$ and positive numbers $\varepsilon_{1}<\eta, \varepsilon_{2}<\varepsilon_{1}, \varepsilon_{3}$ and $\varepsilon_{4}$ such that, if $u \in T$ and $v \in S$, then

(i) $d(u, v)<\varepsilon_{1}$ implies $d(u, v t)>\varepsilon_{1}$ for all $t$ with $\delta \leq|t| \leq \zeta$;

(ii) $d(u, v)<\varepsilon_{2}$ implies $d\left(\phi u, v_{1}\right)<\varepsilon_{1}$;

(iii) $d(u, v) \geq \varepsilon_{2}$ implies $d(u, v t)>\varepsilon_{3}$ for $|t|<\delta$ (which is possible as $\delta<\zeta$ );

(iv) if $a, b \in X$ and $d(a, b)<\varepsilon_{4}$ then $d(a t, b t)<\varepsilon_{1}$ for $|t| \leq \alpha$.

(a) Suppose that for all $i$ for which we have $t_{i}$ and $s_{i}$ defined we have $\left|f\left(t_{i}\right)-s_{i}\right|<\delta$. Choose $j$ such that $d\left(\phi^{i} x, y_{i}\right)>\eta$. Now $d\left(x t_{j}, y s_{j}\right)>\eta>\varepsilon_{1}>\varepsilon_{2}$ so $d\left(x t_{j}, y f\left(t_{j}\right)\right)>\varepsilon_{3}$ by (iii).

(b) Suppose $j$ is the first integer (say positive) such that $\left|f\left(t_{j}\right)-s_{j}\right| \geq \delta$. We may assume that $d\left(\phi^{i} x, y_{i}\right)<\varepsilon_{2}$ for $0 \leq i<j$ (as otherwise we can guarantee separation by $\varepsilon_{3}$ using the argument of (a) again) so that $d\left(\phi^{i} x, y_{j}\right)<\varepsilon_{1}$ by (ii).

(b1) Suppose $t=s_{j}-f\left(t_{j}\right) \geq \delta$. If $f\left(t_{j}\right) \geq s_{j-1}-\delta$, then

$$
\delta \leq t \leq s_{i}-s_{j-1}+\delta<\alpha+\rho+\delta<\zeta
$$

and now

$$
d\left(x t_{j}, y f\left(t_{j}\right)\right)=d\left(x t_{j}, y\left(s_{j}-t\right)\right)>\varepsilon_{1}
$$

by (i). But if $f\left(t_{j}\right)<s_{j-1}-\delta$ then as $f\left(t_{j-1}\right)>s_{j-1}-\delta$ we can find $t^{\prime}$ with $t_{j-1}<t^{\prime}<t_{j}$ and $f\left(t^{\prime}\right)=s_{j-1}-\delta$. Let $\xi=t^{\prime}-t_{j-1}>0$. Then

$$
d\left(x t_{j-1}, y s_{j-1}\right)<\varepsilon_{2}<\varepsilon_{1}
$$

so

$$
d\left(x t_{j-1}, y\left(s_{j-1}-\delta-\xi\right)\right)>\varepsilon_{1}
$$

(since $\delta \leq \delta+\xi<\zeta$ ) by (i). Thus

$$
d\left(x t^{\prime}, y f\left(t^{\prime}\right)\right)=d\left(x\left(t_{j-1}+\xi\right), y\left(s_{j-1}-\delta\right)\right)>\varepsilon_{4}
$$

by (iv).

(b2) Suppose $t=f\left(t_{j}\right)-s_{j} \geq \delta$. Now $f\left(t_{j}\right) \geq s_{j}+\delta$ and $f\left(t_{j-1}\right) \leq s_{j-1}+\delta<s_{j}+\delta$ thus there is a $t^{\prime}$ with $t_{j-1}<t^{\prime} \leq t_{j}$ and $f\left(t^{\prime}\right)=s_{j}+\delta$. Let $\xi=t_{j}-t^{\prime} \geq 0$. Now $d\left(x t_{j}, y s_{j}\right)<\varepsilon_{1}$ implies $d\left(x t_{j}, y s_{j}(\delta+\xi)\right)>\varepsilon_{1}$ by (i) thus

$$
d\left(x t^{\prime}, y f\left(t^{\prime}\right)\right)=d\left(x\left(t_{j}-\xi\right), y\left(s_{j}+\delta\right)\right)>\varepsilon_{4}
$$

by (iv).

In summary, if $\varepsilon^{\prime}=\min \left(\varepsilon_{2}, \varepsilon_{3}, \varepsilon_{4}\right)$ then, for some $t, d(x t, y f(t))>\varepsilon^{\prime}$.

Now suppose $x$ and $y$ are arbitrary points of $X$. Choose $\delta_{1}>0$ and $\varepsilon_{5}>0$ such that $d(x, y)<\varepsilon_{5}$ implies

(i) $d(x t, y s)<\varepsilon^{\prime}$ (where $t$ is the smallest positive number such that $x t \in T^{+}$and $\left.y s=P_{\rho}(y t)\right)$, and $|t-s|<\frac{1}{16} \delta_{1}$; 
(ii) $d(x w, y v)<\varepsilon^{\prime}$ for all $w$ and $v$ with $|w| \leq \delta_{1}+\alpha,|v| \leq \alpha+\delta_{1}$ and $|v-w|<\delta_{1}$. Next let $\varepsilon_{6}>0$ be such that if $d(x, y) \leq \varepsilon_{6}$ then $d\left(x t^{\prime}, y\left(t^{\prime}+t\right)\right)>\varepsilon_{6}$ for all $\frac{1}{16} \delta_{1} \leq|t| \leq$ $\zeta$ and $\left|t^{\prime}\right|<\alpha$. Finally, choose $\varepsilon_{7}>0$ such that $\varepsilon_{5}>\varepsilon_{7}$ and the set of all points in $B_{\varepsilon_{7}}(x)$ which project onto $x t$ is contained in $x\left(-\varepsilon_{5}, \varepsilon_{5}\right)$.

Now suppose $d(x, y)<\varepsilon_{7}$ and $y \notin x\left(-\varepsilon_{5}, \varepsilon_{5}\right)$.

(a) Suppose $|f(t)-s|<\frac{1}{8} \delta_{1}$. Choose $\delta^{\prime}<\frac{1}{4} \delta_{1}$ such that $\left|f\left(t+t^{\prime}\right)-s\right|<\frac{1}{4} \delta_{1}$ for all $\left|t^{\prime}\right| \leq \delta^{\prime}$. Define a continuous real-valued function $g$ such that $g\left(t^{\prime}\right)=f\left(t+t^{\prime}\right)-s$ for all $t^{\prime}$ with $\left|t^{\prime}\right| \geq \delta^{\prime}$ and otherwise by linearity so that $g(0)=0$. Thus

$$
\left|g\left(t^{\prime}\right)-t^{\prime}\right| \leq \frac{1}{4} \delta_{1}+\delta^{\prime}<\frac{1}{2} \delta_{1} \quad \text { for }\left|t^{\prime}\right|<\delta^{\prime}
$$

Thus

$$
d\left(x\left(t+t^{\prime}\right), y\left(s+g\left(t^{\prime}\right)\right)\right)<\varepsilon^{\prime} \quad \text { for }\left|t^{\prime}\right|<\delta^{\prime}
$$

by (i) and (ii). Now by the first part of the proof we can find $t_{0}$ such that $d\left((x t) t_{0},(y s) g\left(t_{0}\right)\right)>\varepsilon^{\prime}$ and by the above construction $\left|t_{0}\right|$ must be greater than $\delta^{\prime}$ i.e.

$$
d\left(x\left(t+t_{0}\right), y f\left(t+t_{0}\right)\right)>\varepsilon^{\prime} .
$$

(Note that $x t \neq y s$ by the choice of $\varepsilon_{7}$.)

(b) If $|f(t)-s| \geq \frac{1}{8} \delta_{1}$ then $|f(t)-t| \geq \frac{1}{16} \delta_{1}$ and so, if we choose $t^{\prime} \leq t$ such that $\left|f\left(t^{\prime}\right)-t^{\prime}\right|=\frac{1}{16} \delta_{1}$, then $d\left(x t^{\prime}, y f\left(t^{\prime}\right)\right)=d\left(x t^{\prime}, y\left(t^{\prime} \pm \delta_{1} / 16\right)\right)>\varepsilon_{6}$ unless $d(x, y)>\varepsilon_{6}$.

It finally follows that $\min \left(\varepsilon^{\prime}, \varepsilon_{6}, \varepsilon_{7}\right)$ is an expansive constant corresponding to $\varepsilon_{5}$.

(2.8) Definition. Suppose that $(X, \mathbb{R})$ is real expansive and that collections $\mathscr{S}$ and $\mathscr{T}$ are constructed as in (2.1) to (2.5). Let $\xi<\varepsilon$ be such that theorem 2.7 holds for $\xi$. Then any $\eta$ with $\xi>\eta>0$ such that $\eta$ is less than the $\delta$ corresponding to $\xi$ given by lemma 2.6 (iii) is called an expansive constant (corresponding to $\mathscr{P}, \mathscr{T}$ and $\xi$ ).

(2.9) LEMMA. Suppose $\left\{x^{n}\right\}$ and $\left\{y^{n}\right\}$ are sequences of points in T and $S$ respectively, $x^{n} \rightarrow x, y^{n} \rightarrow y$ and each $y_{k}^{n}$ is well defined relative to $x^{n}$ for each $k$. Suppose that for some integer $k$ we have $\phi^{k} x^{n} \rightarrow \phi^{l_{k}} x$ for some integer $l_{k}$. Then $y_{k}^{n} \rightarrow y_{l_{k}}^{x}$.

Proof. First note that, if $\left\{\phi^{k} x^{n}\right\}$ converges, it must converge to a point on the $\phi$ orbit of $x$. For if $\phi^{k} x^{n}=x^{n} t_{n},\left\{t_{n}\right\}$ is bounded and we can find a subsequence $t_{n_{i}} \rightarrow t$ (say). Thus $x^{n_{i}} t_{n_{i}} \rightarrow x t$ so $\phi^{k} x^{n} \rightarrow x t \in T$.

Now choose any convergent subsequence of $\left\{y_{k}^{n}\right\}$ and a subsequence of this subsequence (if necessary) such that $\left\{\phi^{j} x^{n}\right\}$ converges for each integer $0 \leq j \leq k$ (denoting these subsequences by $\left\{x^{n}\right\}$ and $\left\{y^{n}\right\}$ again and assuming $k \geq 0$ for convenience). We complete the proof by showing that $y_{k}^{n} \rightarrow y_{l_{k}}^{x}$. Suppose $\phi^{i} x^{n} \rightarrow$ $\phi^{l_{i}} x(0 \leq j \leq k)$ and suppose that for some integer $m(0 \leq m<k) y_{i}^{n} \rightarrow y_{l_{i}}^{x}$ for all $0 \leq i \leq$ $m$. Let $\phi^{m+1}\left(x^{n}\right)=\phi^{m}\left(x^{n}\right) t_{n}$ and $y_{m+1}^{n}=y_{m}^{n} s_{n}$ where $s_{n}=t_{n}+\eta_{n}$ and $\left|\eta_{n}\right|<\rho$. Now as both $\left\{\phi^{m+1}\left(x^{n}\right)\right\}$ and $\left\{\phi^{m}\left(x^{n}\right)\right\}$ converge, $t_{n} \rightarrow t$, say (since $2 \alpha<\zeta$ ), and thus $s_{n} \rightarrow s$ and $\eta_{n} \rightarrow \eta$ (since $2 \rho<\zeta$ ). Thus $y_{m+1}^{n} \rightarrow y_{l_{m}}^{x} s=y_{l_{m+1}}$, say. Now

$$
d\left(\phi^{l_{m+1}} x, y_{l_{m+1}}\right) \leq d\left(\phi^{l_{m+1}} x, \phi^{m+1} x^{n}\right)+d\left(\phi^{m+1} x^{n}, y_{m+1}^{n}\right)+d\left(y_{m+1}^{n}, y_{l_{m+1}}\right)
$$

so that letting $n \rightarrow \infty$ gives

$$
d\left(\phi^{l_{m+1}} x, y_{l_{m+1}}\right) \leq \varepsilon
$$


Also $t \leq \alpha$ so

$$
y_{l_{m+1}}^{x}=P_{\rho}\left(y_{l_{m}}^{x} t\right)
$$

by lemma 2.6 (i) and

$$
y_{l_{m+1}}=y_{l_{m}}^{x}(t+\eta)=P_{\rho}\left(y_{l_{m}}^{x} t\right)
$$

Thus $y_{l_{m+1}}=y_{l_{m+1}}^{x}$ as required. Since the induction hypothesis holds trivially for $m=0$, it holds for all integers up to $k$.

(2.10) Corollary. Let $(X, \mathbb{R})$ be real expansive, let $\eta$ be an expansive constant and $y \in W_{\eta}^{s}(x)$. Then $d\left(\phi^{n} x, y_{n}\right) \rightarrow 0$ as $n \rightarrow \infty$. Similarly, if $y \in W_{\eta}^{u}(x)$, then $d\left(\phi^{-n} x, y_{-n}\right) \rightarrow 0$ as $n \rightarrow \infty$.

Proof. If not, we can find points $x, y \in X$ with $y \in W_{\eta}^{s}(x), \delta>0$, and an increasing sequence of integers $\left\{n_{i}\right\}$ such that $d\left(\phi^{n_{i}} x, y_{n_{i}}\right) \geq \delta$ for each $i$ and $\phi^{n_{i}} x \rightarrow a$ and $y_{n_{i}} \rightarrow b$. Then $a \in T, b \in S$ and $a \neq b$. Now let $k$ be an arbitrary integer. Choosing a subsequence if necessary, $\phi^{n_{i}+k} x \rightarrow \phi^{l_{k}} a$ (say) and thus, by lemma 2.9, $y_{n_{i}+k} \rightarrow b_{l_{k}}^{a}$. Note that if $i$ is large enough so that $n_{i}+k>0$, then $y_{n_{i}+k}$ is well-defined. But now $d\left(\phi^{l_{k}} a, b_{l_{k}}^{a}\right) \leq \eta$ for all $k$, which contradicts the choice of $\eta$ (theorem 2.7).

(2.11) Corollary (Uniform expansiveness). Let $\eta$ be an expansive constant. Then for all $0<\delta<\eta$ there is an integer $N(\delta)>0$ such that, if $x \in T, y \in S$ and $d(x, y) \geq \delta$, then $d\left(\phi^{i} x, y_{i}\right)>\eta$ for some integer $i$ with $|i|<N(\delta)$.

Proof. Let $\eta$ be an expansive constant and suppose the statement does not hold. Then there is a $\delta>0$ such that for every integer $N>0$ we can find points $x^{N} \in T$ and $y^{N} \in S$ with $d\left(x^{N}, y^{N}\right) \geq \delta$ but $d\left(\phi^{i} x^{N}, y_{i}^{N}\right) \leq \eta$ for all $|i| \leq N$. Choose an integer $k$ and assume $x^{N} \rightarrow x, y^{N} \rightarrow y$ and $\phi^{k} x^{N} \rightarrow \phi^{l_{k}} x$, say. Then by lemma $2.9 y_{k}^{N} \rightarrow y_{l_{k}}^{x}$. But now

$$
d\left(\phi^{l_{k}} x, y_{l_{k}}^{x}\right) \leq d\left(\phi^{I_{k}} x, \phi^{k} x^{N}\right)+d\left(\phi^{k} x^{N}, y_{k}^{N}\right)+d\left(y_{k}^{N}, y_{l_{k}}^{x}\right)
$$

and so letting $N \rightarrow \infty$ we obtain

$$
d\left(\phi^{l_{k}} x, y_{l_{k}}^{x}\right) \leq \eta
$$

This contradicts the fact that $x \neq y$.

(2.12) LEMMA. If $\eta>0$ is sufficiently small, there is a $\delta>0$ such that if $p \in W_{\delta}^{s}(x)$ and $q \in W_{\delta}^{s}(x)$ then $p^{\prime} \in W_{\eta}^{s}\left(q^{\prime}\right)$ where $q^{\prime}=q c$ and $p^{\prime}=P_{\rho}(p c)$ and $c$ is the smallest non-negative number such that $q c \in T^{+}$.

Proof. If $\delta<\frac{1}{2} \varepsilon$ then for any $a, b \in S, d(a, b)<2 \delta$ and $0<t \leq 3 \alpha$, if $a t \in T$ then $b t \in D_{\rho}$. Choose $\delta$ small enough so that $d\left(a t, P_{\rho}(b t)\right)<\eta$. Now choose an integer $k \geq 0$ and assume $\phi^{k} q^{\prime}$ lies on the orbit of $q$ at or after $q_{i}^{x}$ and before $q_{i+1}^{x}$. Then as $d\left(q_{i}^{x}, p_{i}^{x}\right)<2 \delta$ we have

$$
d\left(\phi^{k} q^{\prime}, P_{\rho}\left(p_{i}^{x} s\right)\right)<\eta,
$$

where $\phi^{k} q^{\prime}=q_{i}^{x} s$. We shall show by induction that $P_{\rho}\left(p_{i}^{x} s\right)=p_{k}^{\prime q^{\prime}}$.

First consider $k=0$. If $q^{\prime}$ lies between $q_{i}^{x}$ and $q_{i+1}^{x}$ then $q^{\prime}=q_{i}^{x}\left(c-s+\eta_{1}\right)$, where $\phi^{i} x=x s$, and $p^{\prime}=p_{i}^{x}\left(c+\eta_{2}-s+\eta_{3}\right)$, where $\left|\eta_{1}\right|,\left|\eta_{2}\right|,\left|\eta_{3}\right|<\rho$. Now

$$
P_{\rho}\left(p_{i}^{x}\left(c-s+\eta_{1}\right)\right)=p_{i}^{x}\left(c-s+\eta_{1}+\eta_{4}\right)=p^{\prime}\left(-\eta_{2}-\eta_{3}+\eta_{1}+\eta_{4}\right) .
$$


where $\left|\eta_{4}\right|<\rho$. Since $5 \rho<\zeta$, this point is $p^{\prime}$ and the claim holds. So suppose it holds for some $k \geq 0$. Let $\phi^{k} q^{\prime}$ lie between $q_{i}^{x}$ and $q_{i+1}^{x}$, let $\phi^{k+1} q^{\prime}$ lie between $q_{i}^{x}$ and $q_{j+1}^{x}$ for some $j \geq i$ and suppose $\phi^{k} q^{\prime}=q_{i}^{x} s$ and $\phi^{k+1} q^{\prime}=q_{i}^{x}$. Let $t_{i j}>0$ be such that $\left(\phi^{i} x\right) t_{i j}=\phi^{i} x$. Note that if $u$ is such that $q_{j}^{x}=q_{i}^{x} u$ then $0 \leq u \leq \alpha+\alpha+\rho-\beta \leq 2 \alpha$ and $0 \leq t_{i j} \leq 2 \alpha+\rho$ by lemma 2.6 (ii). Now

and

$$
\phi^{k+1} q^{\prime}=q_{i}^{x}\left(t_{i j}+t+\eta_{i}\right)
$$

$$
P_{\rho}\left(p_{i}^{x} t\right)=p_{i}^{x}\left(t_{i j}+\eta_{2}+t+\eta_{3}\right)
$$

where $\left|\eta_{1}\right|,\left|\eta_{2}\right|,\left|\eta_{3}\right|<\rho$. Also

$$
\begin{aligned}
p_{k+1}^{\prime q^{\prime}} & =p_{k}^{\prime q^{\prime}}\left(t_{i j}+t+\eta_{1}-s+\eta_{4}\right)=P_{\rho}\left(p_{i}^{x} s\right)\left(t_{i j}+t-s+\eta_{1}+\eta_{4}\right) \\
& =p_{i}^{x}\left(t_{i j}+t+\eta_{1}+\eta_{4}+\eta_{5}\right),
\end{aligned}
$$

where $\left|\eta_{4}\right|,\left|\eta_{5}\right|<\rho$. As $5 \rho<\zeta$ and $P_{\rho}\left(p_{j}^{x} t\right)$ and $p_{k+1}^{\prime q^{\prime}}$ belong to the same set in $\mathscr{S}$, $P_{\rho}\left(p_{i}^{x_{t}}\right)=p_{k+1}^{\prime q^{\prime}}$ as required.

\section{One dimensional minimal sets of real-expansive flows}

In this section we discuss conditions under which the minimal sets of a real-expansive flow are one dimensional. The conditions cover the known examples of such flows. Throughout we shall assume that we have constructed $\mathscr{S}$ and $\mathscr{T}$ and chosen $\rho$ as in $\S 2$ and that $\eta$ is an expansive constant if the flow is real-expansive.

Our first lemma is closely analogous to lemma 3 of [5] and guarantees the existence of orbits asymptotic in our sense. Let $S_{\delta}(x)=\{a \in X ; d(x, a)=\delta\}, B_{\delta}(x)=$ $\{a \in X, d(x, a) \leq \delta\}, \Sigma_{\delta}^{s}(x)$ and $\Sigma_{\delta}^{u}(x)$ be the connected components of a point $x \in T$ in $W_{\eta}^{s}(x) \cap B_{\delta}(x)$ and $W_{\eta}^{u}(x) \cap B_{\delta}(x)$ respectively, and let $\Sigma_{\delta}(x)$ be the connected component of $x$ in $B_{\delta}(x) \cap S$.

(3.1) Lemma. Suppose $(X, \mathbb{R})$ is a real flow and $X$ is not one dimensional. There exists $r$ with $\eta>r>0$ such that, for any $\delta$ with $0<\delta<r$, there is $T \in \mathscr{T}$ and $a \in T$ such that either $\Sigma_{\delta}^{s}(a) \cap S_{\delta}(a) \neq \varnothing$ or $\Sigma_{\delta}^{u}(a) \cap S_{\delta}(a) \neq \varnothing$.

Proof. Choose a real number $q$ so that the map $a \rightarrow a_{1}^{z}$ is continuous on $B_{q}(z) \cap S$ if $z \in T$. Clearly we can find $T \in \mathscr{T}$ with $\operatorname{dim}(T)>0$ and thus $x \in T$ and $q>r>0$ such that $\Sigma_{r}(x) \cap S_{r}(x) \neq \varnothing$. Suppose that for some $0<\delta<r$ we have $\Sigma_{\delta}^{u}(y) \cap S_{\delta}(y)=$ $\varnothing$ for all $y \in T$. We construct a sequence of compact connected sets $\left\{\Lambda_{n}\right\}$ with each $\Lambda_{n} \subset S$, a sequence $\left\{x_{n}\right\}$ of points of $T$ with $x_{n} \in \Lambda_{n}$, and a sequence of positive integers $\left\{m_{n}\right\}$ such that:

(1) $\phi^{m_{n}} x_{n+1}=x_{n}$;

(2) $\left(\Lambda_{n+1}\right)_{m_{n}}^{x_{n+1} \subset \Lambda_{n}}$;

(3) $\Lambda_{n} \cap S_{\delta}\left(x_{n}\right) \neq \varnothing$;

(4) $\left(\Lambda_{n}\right)_{i}^{x_{n}} \subset B_{\delta}\left(\phi^{j} x_{n}\right)$ if $0 \leq j<m_{n-1}$.

Take $\Lambda_{0}=\Sigma_{\delta}(x)$ and $x_{0}=x$. Suppose $\Lambda_{0}, \ldots, \Lambda_{n}$ and $x_{0}, \ldots, x_{n}$ and $m_{0}, \ldots, m_{n-1}$ have been constructed. Let $\Gamma$ be the connected component of $x_{n}$ in $B_{\delta}\left(x_{n}\right) \cap \Lambda_{n}$. Then $\Gamma \subset S$ and $\Gamma \cap S_{\delta}\left(x_{n}\right) \neq \varnothing$. Also $\Gamma \not \subset W_{n}^{u}\left(x_{n}\right)$ and, if $y \in \Gamma-W_{n}^{u}\left(x_{n}\right)$, there is an integer $m_{y}>\theta$ such that

$$
d\left(\phi^{-m_{y}} x_{n}, y_{-m_{y}}\right)>\delta .
$$


Define

$$
m_{n}=\min \left\{m_{y} ; y \in \Gamma \operatorname{such} \text { that } m_{y} \text { exists }\right\}
$$

and so

$$
\sup _{z \in \Gamma} d\left(\phi^{-i} x_{n}, z_{-j}\right) \leq \delta \text { for all } 0 \leq j<m_{n} \text {. }
$$

Now let $\Lambda_{n+1}$ be the connected component of $x_{n+1}=\phi^{-m_{n}}\left(x_{n}\right)$ in $B_{\delta}\left(x_{n+1}\right) \cap(\Gamma)_{-m_{n}}^{x_{n}}$. $(\Gamma)_{-m_{n}}^{x_{n}}$ is a compact connected set because the map $a \rightarrow a_{m}^{z}$ (relative to a fixed $z \in T)$ is continuous for $-m_{n} \leq m \leq 0$ by the choice of $q$. Now for some $y \in \Gamma$, $d\left(x_{n+1}, y_{-m_{n}}\right)>\delta$ so that $\Lambda_{n+1} \cap S_{\delta}\left(x_{n+1}\right) \neq \varnothing$. Thus (1), (2) and (3) are satisfied and (4) follows by the choice of $m_{n}$.

Now choose a subsequence of $\left\{x_{n}\right\}$ such that $x_{n} \rightarrow a$ and, using this subsequence, also assume that $\Lambda=\lim _{2^{x}} \Lambda_{n}$ exists.

So $\Lambda$ consists of points $p$ such that for some subsequence $\left\{p_{n_{i}}\right\}$ with $p_{n_{i}} \in \Lambda_{n_{i}}, p_{n_{i}} \rightarrow p$. Clearly $\Lambda$ is a compact connected set, $\Lambda \subset B_{\delta}(a)$ and $\Lambda \cap S_{\delta}(a) \neq \varnothing$. We shall show that $\Lambda \subset W_{\eta}^{s}(a)$. Let $k>0$ and choose $n$ sufficiently large so that we can write $k=m_{n-1}+m_{n-2}+\cdots+m_{n-i}+l$, where $0 \leq l<m_{n-i-1}$. Now $\phi^{k}\left(x_{n}\right)=\phi^{l}\left(x_{n-i}\right)$ and, if $p \in \Lambda_{n}$, then $p_{k}^{x_{n}}=g \imath^{x_{n-i}}$ where $g \in \Lambda_{n-i}$.

Now suppose $p \in \Lambda$. Choose a subsequence $\left\{x_{n_{j}}\right\}$ such that $p_{n_{j}} \rightarrow p\left(p_{n_{j}} \in \Lambda_{n_{j}}\right)$ and $\phi^{k}\left(x_{n_{i}}\right) \rightarrow \phi^{l_{k}} a$ for some integer $l_{k}$. By lemma 2.9

$$
\left(p_{n_{j}}\right)_{k}^{x_{n_{i}} \rightarrow p_{l_{k}}^{a}} \text { and } d\left(\left(p_{n_{j}}\right)_{k}^{x_{n_{i}}}, \phi^{k}\left(x_{n_{j}}\right)\right)=d\left(g_{l^{n_{j-i}}}^{x_{n}}, \phi^{l}\left(x_{n_{i-i}}\right)\right) \leq \delta,
$$

where $g$ and $l$ are suitably chosen as above and we have used (4) for $\Lambda_{n_{j-i}}$. Thus $d\left(\phi^{k_{k}} a, p_{l_{k}}^{a}\right) \leq \delta$. We now only have to ensure that $r$ (and so $\delta$ ) is small enough so that this implies $d\left(\phi^{i} a, p_{i}^{a}\right) \leq \eta$ for all $i \geq 0$ using lemma 2.6 (iii).

(3.2) Remark. If $(X, \mathbb{R})$ is real expansive, then it has a pair of negatively or positively weakly asymptotic points (i.e. points $a \neq b \in X$ such that $d\left(\phi^{n} a, b_{n}\right) \rightarrow 0$ as $n \rightarrow-\infty$ or $n \rightarrow+\infty$ ). For if $X$ is one dimensional this follows by the remark after definition 2.5 and theorem 6 of [3]. Otherwise, the result follows by the above lemma and corollary 2.10. In fact, one can choose $a$ and $b$ to be on distinct orbits.

(3.3) LemMA. Let $(X, \mathbb{R})$ be real expansive and let $\eta$ be an expansive constant. Then given $0<\eta^{\prime}<\eta$ there exists a $\delta_{0}>0$ such that

$$
W_{\eta}^{s}(x) \cap B_{\delta}(x)=W_{\eta^{\prime}}^{s}(x) \cap B_{\delta}(x)
$$

for all $x \in T^{+}$and $0<\delta<\delta_{0}$.

Proof. If not, we can find sequences $\left\{x_{n}\right\} \subset T^{+}$and $\left\{y_{n}\right\} \subset S^{+}$such that $d\left(x_{n}, y_{n}\right) \rightarrow 0$, $y_{n} \in W_{\eta}^{s}\left(x_{n}\right)$ and an increasing sequence of positive integers $\left\{m_{n}\right\}$ such that $d\left(\phi^{m_{n}} x_{n},\left(y_{n}\right)_{m_{n}}^{x_{n}}\right)>\eta^{\prime}$. Let $\phi^{m_{n}} x_{n} \rightarrow x$ and $\left(y_{n}\right)_{m_{n}}^{x_{n}} \rightarrow y$, then $d(x, y) \geq \eta^{\prime}$ and, for each integer $k>0$, we can assume $\phi^{k} \phi^{m_{n}} x_{n} \rightarrow \phi^{l_{k}} x$ by successively choosing an appropriate subsequence for the $k+1$ case from the subsequence for the $k$ case; similarly for $k<0$. Thus

$$
\left(y_{n}\right)_{m_{n+k}}^{x_{n}}=\left(\left(y_{n}\right)_{m_{n}}^{x_{n}}\right)_{k}^{\phi m_{n} x_{n}} \rightarrow y_{l_{k}}^{x}
$$


by lemma 2.9 . Thus

$$
d\left(\phi^{l_{k}} x, y_{l_{k}}^{x}\right) \leq \eta
$$

for all $k$ which contradicts the fact that $\eta$ is an expansive constant.

(3.4) LEMMA. Let $(X, \mathbb{R})$ be a real-expansive flow. Then there is an expansive constant $\eta$ such that, for all $\delta^{\prime}$ sufficiently small, there is $a \delta>0$ and an integer $N(\delta)$ such that, if $x \in T$ and $\Lambda \subset W_{\eta}^{s}(x)$ is a compact connected set containing $x$ and intersecting $S_{\delta}(x)$, then there exists an integer $m$ with $0<m<N(\delta)$, points $v, w \in S^{+}$ and compact connected sets $\Lambda_{v}$ and $\Lambda_{w}$ contained in $S^{+}$such that:

(a) $v^{\prime} \in \Lambda_{v} \cap T^{+}, v=v^{\prime}(-t) \in \Lambda_{-m}^{x}($ for some $0 \leq t<\alpha)$ and $P_{\rho}\left(\Lambda_{v}(-t)\right) \subset \Lambda_{-m}^{x} \cap$ $B_{\delta^{\prime}}(v) \subset W_{\eta}^{s}\left(\phi^{-m} x\right)$, and a similar statement holds for $w$ and $\Lambda_{w}$;

(b) $\Lambda_{v} \cap S_{\delta}\left(v^{\prime}\right) \neq \varnothing$ and $\Lambda_{w} \cap S_{\delta}\left(w^{\prime}\right) \neq \varnothing$;

(c) $d\left(B_{\delta^{\prime}}(v), B_{\delta^{\prime}}(w)\right)>\delta$;

(d) $\Lambda_{v} \subset W_{\eta}^{s}\left(v^{\prime}\right) \cap B_{\delta}\left(v^{\prime}\right)$ and $\Lambda_{w} \subset W_{\eta}^{s}\left(w^{\prime}\right) \cap B_{\delta}\left(w^{\prime}\right)$.

Proof. Choose $\eta^{\prime}$ an expansive constant corresponding to the $\eta$ of lemma 2.12 and $\eta<\eta^{\prime}$ corresponding to the $\delta$ of that lemma. Let

$$
\eta_{1}=\inf \left\{d(x, y) ; x \in T^{+}, y \in S^{+}, d(x, y) \leq \eta \quad \text { and } \quad d\left(\phi^{-1} x, y_{-1}^{x}\right)>\eta\right\}
$$

and

$$
\eta_{2}=\inf \left\{d\left(x t, P_{\rho}(y t)\right) ; x, y \in S^{+}, x t \in T^{+} \quad \text { and } \quad 0 \leq t \leq \alpha, \quad \text { and } \quad \eta \geq d(x, y) \geq \eta_{1}\right\} \text {. }
$$

Choose $\delta$ and $\delta^{\prime}$ such that $\delta^{\prime}<\frac{1}{3} \eta_{1}, \delta<\frac{1}{2} \eta_{2}, \delta<\delta^{\prime}<\eta$. If $a, b \in S^{+}$and $0 \leq t<\alpha$ such that $a t \in T^{+}$then, if $d\left(a t, P_{\rho}(b t)\right) \leq \delta$, we have $d(a, b) \leq \delta^{\prime}$, and finally $\delta<\delta_{0}$, where $\delta_{0}$ is given by lemma 3.3 (interchanging the roles of $\eta$ and $\eta^{\prime}$ ). Now let $N(\delta)$ be the number given by corollary 2.11 corresponding to $\delta$ and $\eta$. Since $S_{\delta}(x) \cap \Lambda \neq$ $\varnothing$, by that corollary we can find $m$ with $0 \leq m<N(\delta)$ such that

$$
\sup \left\{d\left(\phi^{-(m+1)} x, z_{-(m+1)}\right) ; z \in \Lambda\right\}>\eta \quad \text { and } \sup \left\{d\left(\phi^{-j} x, z_{-j}\right) ; z \in \Lambda\right\} \leq \eta
$$

for $0 \leq j \leq m$. Thus

$$
\Lambda_{-m}^{x} \subset W_{\eta}^{s}\left(\phi^{-m} x\right) .
$$

Also since the diameter of $\Lambda_{-m}^{x}$ is at least $\eta_{1}$, we can find points $v, w \in \Lambda_{-m}^{x}$ such that $(c)$ holds. If we now construct $v^{\prime}$ and project $\Lambda_{-m}^{x}$ onto the section containing $v^{\prime}$, we obtain a compact connected set with diameter at least $\eta_{2}$. Let $\Lambda_{v}$ be the connected component of this set in $B_{\delta}\left(v^{\prime}\right)$ intersected with the set. Do the same construction for $W$. We then have $(a)$ and $(b)$. Now by lemma 2.12 we have $\Lambda_{v} \subset W_{\eta^{\prime}}^{s}\left(v^{\prime}\right)$ and by lemma 3.3

$$
\Lambda_{v} \subset W_{\eta}^{s}\left(v^{\prime}\right) \cap B_{\delta}\left(v^{\prime}\right)
$$

as $\delta<\delta_{0}$. This gives $(d)$.

(3.5) Definition. A flow $(X, \mathbb{R})$ is said to have a spiral orbit at $x$ if $x t \in W_{\eta}^{s}(x)$ for some $t>0$ and if $d\left(\phi^{n} x,(x t)_{n}\right) \rightarrow 0$.

(3.6) THEOREM. Let $(X, \mathbb{R})$ be a real-expansive minimal flow which has no spiral orbits. Then $X$ is one dimensional (and so a suspension of a subshift [3], [4]).

Proof. Let $\boldsymbol{\eta}$ be an expansive constant small enough to satisfy the requirements of lemmas 2.12 (i.e. $\eta<\delta$ ) and 3.4. Choose $\delta<r$ of lemma 3.1 and corresponding to the 
$\eta$ of lemma 3.4 and choose $N(\delta)$ as in that lemma. Now choose a positive integer $N$ with $N>(1+N(\delta))(\alpha+\rho) / \beta$. Let (primed points as in lemma 3.4)

$$
\begin{aligned}
& r_{1}=\inf \left\{d\left(\phi^{-i} x^{\prime}, \phi^{-i} y^{\prime}\right) ; d(x, y) \geq \delta, x, y \in S^{+}, 0 \leq i, j \leq N\right. \\
& \text { and there exists } \left.z \in T^{+} \text {with } x \in W_{\eta}^{s}(z) \text { and } y \in W_{\eta}^{s}(z)\right\} .
\end{aligned}
$$

First suppose $r_{1}=0$. In that case we can find sequences $\left\{x_{n}\right\}$ and $\left\{y_{n}\right\}$ in $S^{+}$and $\left\{z_{n}\right\}$ in $T^{+}$such that $d\left(x_{n}, y_{n}\right) \geq \delta, x_{n} \in W_{\eta}^{s}\left(z_{n}\right), y_{n} \in W_{\eta}^{s}\left(z_{n}\right)$ and $d\left(\phi^{-i} x_{n}^{\prime}, \phi^{-i} y_{n}^{\prime}\right) \rightarrow 0$ for fixed integers $i, j$ with $0 \leq i, j \leq N$. Let $x_{n} \rightarrow x, y_{n} \rightarrow y, z_{n} \rightarrow z$. By lemma 2.9 $x, y \in W_{\eta_{1}}^{s}(z)\left(\eta_{1}<\delta\right.$ of lemma 2.12) and so $x^{\prime} \in W_{\eta_{2}}^{s}\left(y^{\prime}\right)$, where $x^{\prime}, y^{\prime}$ and $\eta_{2}$ are given as in lemma 2.12. Further, let $x_{n}^{\prime} \rightarrow x^{\prime \prime}, y_{n}^{\prime} \rightarrow y^{\prime \prime}, \phi^{-i} x_{n}^{\prime} \rightarrow \phi^{-l_{i}} x^{\prime \prime}$ and $\phi^{-i} y_{n}^{\prime} \rightarrow$ $\phi^{-l_{i}} y^{\prime \prime}$. Clearly $x^{\prime \prime}=\phi^{l_{i}-l_{i}} y^{\prime \prime}$. Now if $l_{j}=l_{i}$, then $x^{\prime \prime}=y^{\prime \prime}$ but, as $2 \alpha<\zeta$, this implies $x=y$, contradicting $d(x, y) \geq \delta$. Now $x^{\prime \prime}=y^{\prime \prime} T$, say, and assume $T>0$. Also $y^{\prime \prime}=y^{\prime} t$ and $t \geq 0$ and $x^{\prime \prime}=x^{\prime}$. Thus $x^{\prime}=y^{\prime}(T+t-s)$ and so, as $y^{\prime}$ cannot lie between $x^{\prime}$ and $x^{\prime \prime}, T+t-s>0$, which means that the flow has a spiral orbit at $y$.

Now suppose $r_{1}>0$. We construct a sequence of compact connected sets $\left\{\Lambda^{n}\right\}$ and points $\left\{x_{n}\right\} \subset T^{+}$with $x_{n} \in \Lambda^{n}$ such that:

(a) $\Lambda^{n} \cap S_{\delta}\left(x_{n}\right) \neq \varnothing$;

(b) $\Lambda^{n} \subset W_{n}^{s}\left(x_{n}\right)$;

(c) for some $m_{n}$ with $0<m_{n}<N(\delta)$

$\left(P_{\rho}\left(\Lambda^{n+1}(-t)\right)\right)_{m_{n}}^{\phi-m_{n}\left(x_{n}\right)} \subset \Lambda_{n} \quad$ for some $|t|<\alpha ;$

(d) $\left(\Lambda^{n}\right)_{-j}^{x_{n}} \cap U=\varnothing$ for $0 \leq j \leq m_{n} \quad(n>0)$,

where $U$ is an open set in some $T^{*}$ with diameter less than $\frac{1}{2} r_{1}$ and such that $U \cap \Sigma_{\delta}^{s}(a)=\varnothing$ and $d(U, S-T)=\tau>0$.

Set $x_{0}=a$ (given by lemma 3.1), $\Lambda^{0}=\Sigma_{\delta}^{s}(a)$ and suppose $\Lambda^{0}, \Lambda^{1}, \ldots, \Lambda^{n}$ and $x_{0}, x_{1}, \ldots, x_{n}$ are constructed. Now applying lemma 3.4 to $\Lambda^{n}$ we obtain $m_{n}, v, w$, $\Lambda_{v}$ and $\Lambda_{w}$. Note that choosing $x_{n+1}$ and $\Lambda^{n+1}$ as $v^{\prime}$ and $\Lambda_{v}$ or $w^{\prime}$ and $\Lambda_{w}$ satisfies $(a),(b)$ and $(c)$. Suppose $U \cap \bigcup_{j=0}^{m_{n}}\left(\Lambda_{v}\right)_{-j}^{v^{\prime}} \neq \varnothing$ and also $U \cap \bigcup_{j=0}^{m_{n}}\left(\Lambda_{w}\right)_{-j}^{w^{\prime}} \neq \varnothing$. Then we can find $p \in \Lambda_{v}$ and $q \in \Lambda_{w}$ with $p_{-j}^{v^{\prime}} \in U$ and $q_{-i}^{w^{\prime}} \in U$ for some $i$ and $j$ with $0 \leq i, j \leq m_{n}$. Let $p^{\prime \prime}$ and $q^{\prime \prime}$ be the first points of the negative orbits of $p$ and $q$ in $\Lambda_{-m_{n}}^{x_{n}}$. Let $p^{\prime}$ and $q^{\prime}$ be the projection of $p^{\prime \prime}$ and $q^{\prime \prime}$ onto $T^{+}$. Thus $d\left(p^{\prime \prime}, q^{\prime \prime}\right) \geq \delta$ by lemma 3.4 (c) and $p^{\prime \prime}, q^{\prime \prime} \in W_{\eta}^{s}\left(\phi^{-m_{n}} x_{n}\right)$. Since $U \subset T^{*}, p_{-i}^{v^{\prime}}=\phi^{-l} p^{\prime}$ (say) and $q_{-i}^{w^{\prime}}=\phi^{-s} q^{\prime}$ (say) and so $d\left(\phi^{-l} p^{\prime}, \phi^{-s} q^{\prime}\right)<\frac{1}{2} r_{1}$. By the choice of $N, 0 \leq l, s \leq N$ which is a contradiction.

So choose $\Lambda^{n+1}$ to be $\Lambda_{v}$ if $U \cap \bigcup_{j=0}^{m_{n}}\left(\Lambda_{v}\right)_{-j}^{v^{\prime}}=\varnothing$ and to be $\Lambda_{w}$ otherwise. We thus have $(d)$ also.

We now use the notation

$$
\left(\Lambda^{n}\right) \sum_{i=0}^{n-1} x_{i}
$$

to mean that we shift the projection of $\Lambda^{n}$ into $\Lambda_{-m_{n-1}}^{n-1} m_{n-1}$ steps relative to $\phi^{-m_{n-1}} x_{n-1}$, then the projection of this set into $\Lambda_{-m_{n-2}}^{n-2}$ is shifted $m_{n-2}$ steps relative to $\phi^{-m_{n-2}} x_{n-2}$ and so on up to the projection in $\Lambda_{-m_{0}}^{0}$ is shifted $m_{0}$ steps relative 
to $\phi^{-m_{0}} x_{0}$. Let

$$
A=\bigcap_{n \geq 1}\left(\Lambda^{n}\right) \sum_{i=0}^{\sum_{i=0}^{n-1} x_{i}} .
$$

Clearly, for any integer $N$, we have

$$
\left(\Lambda^{N}\right) \sum_{i=0}^{\sum_{i=0}^{N-1} x_{i}} \subset\left(\Lambda^{N-1}\right) \sum_{i=0}^{\sum_{i=0}^{N-2} x_{i}}
$$

so that the intersection in the definition of $A$ is nested and thus $A \neq \varnothing$. Let $p \in A$. We complete the proof by showing that there is $K>0$ such that for all $k \geq K$, $p(-k) \notin U$, which contradicts minimality. Inductively construct a sequence of points $\left\{p^{-j}\right\}$ on the orbit of $p$ by $p^{0}=p$ and for $j$ satisfying

$$
\sum_{i=0}^{n-1} m_{i}<j<\sum_{i=0}^{n} m_{i}
$$

(interpret the first sum as 0 if $n=0$ )

$$
p^{-j}=\left(p^{-\sum_{i=0}^{n-1} m_{i}}\right)_{-j+\sum_{i=0}^{n-1} m_{i}}^{x_{n}} \text { and } p^{-\sum_{i=0}^{n} m_{i}}=\left(p^{-\sum_{i=0}^{n-1} m_{i}}\right)_{-m_{n} t}^{x_{n}}
$$

where $t$ is the smallest non-negative number such that this point is in $\Lambda^{n+1}$. It is now clear that

$$
p^{-i} \in\left(\Lambda^{n}\right)_{-j+i{ }^{x_{n}} m_{i}}^{x_{1}}
$$

so that for each $j>m_{0}, p^{-i} \notin U$. Now suppose that the orbit of $p$ intersects $U$ between $p^{-i}$ and $p^{-j-1}$. Make $\varepsilon$ small enough so that, if $d(a, b)<\varepsilon$ and $a t \in T$ for $|t|<\alpha+\rho$, then $d\left(a t, P_{\rho}(b t)\right)<\tau$. Now if $\sum_{i=0}^{n-1} m_{i} \leq j<\sum_{i=0}^{n} m_{i}$, then, by the definition of $p^{-i}$, the orbit of $x_{n}$ also intersects $U$ between

$$
\phi^{-i+\sum_{i=0}^{n-1} m_{i}}\left(x_{n}\right) \text { and } \phi^{-i-1+\sum_{i=0}^{n-1} m_{i}}\left(x_{n}\right)
$$

which is false.

(3.7) Remark. It is by no means obvious that the existence of a spiral orbit leads to a periodic point (as in the discrete case). However, this is the case for suspensions (so we recover Mañé's result [5]) and also for flows which exhibit a strong form of uniform expansiveness which include Axiom A flows (so we recover Bowen's result [2]). It would be interesting to have examples of spiral orbits in a minimal real flow; if the example were also expansive, it would be an example of a minimal expansive flow which does not have a one dimensional phase space (corollary 3.9).

(3.8) LEMMA. Let $(X, \mathbb{R})$ be a minimal flow with a spiral orbit at $x$. Suppose $x T \in W_{\eta}^{s}(x)$ for some $T>0$, and for each $n,\left(\phi^{n} x\right) \bar{t}_{n}=(x T)_{n}$. Then $\bar{t}_{n} \rightarrow \infty$.

Proof. Note that $\bar{t}_{n} \geq \zeta$ for all $n$ so that if $\bar{t}_{n} \rightarrow \infty$ we can find a subsequence $\bar{t}_{n_{i}} \rightarrow \bar{t}>0$. Now $d\left(\phi^{n} x,(x T)_{n}\right) \rightarrow 0$ so if we let $\phi^{n_{i}} x \rightarrow z$ we obtain $d(z, z \bar{t})=0$ and $z$ is a periodic point. 
(3.9) Corollary (Mañé). Let $(X, \mathbb{R})$ be a minimal real-expansive flow which is a suspension. Then $(X, \mathbb{R})$ has no spiral orbits. Thus $(X, \mathbb{R})$ is a suspension of a subshift.

Proof. In a suspension, there are always the same number of returns to the base between $\phi^{n} x$ and $(x T)_{n}$, if $\rho$ is chosen sufficiently small. Thus $\left\{\bar{t}_{n}\right\}$ of lemma 3.8 is bounded.

(3.10) Definition. A real flow $(X, \mathbb{R})$ is said to have property $\left({ }^{*}\right)$ if it has the following property:

For each $\rho_{0}>0$ there is an $\eta>0$ and a sequence of positive real numbers $\left\{a_{n}\right\}$ such that $\sum_{n=1}^{\infty} a_{n}<\infty$ and if $x, y \in S$ with $d(x, y)<\eta$ and for some $|t|<\alpha+2 \rho_{0}$, $d\left(x t, y t\left(-\rho_{0}, \rho_{0}\right)\right)>a_{n}$, then $d\left(\phi^{i} x^{\prime}, y_{i}^{\prime}\right)>\eta$ for some $|i| \leq n$ and $x^{\prime}=x s$, where $s>0$ is the smallest time such that $x s \in T^{+}$and $y^{\prime}=P_{\rho}(y s)$.

(Clearly (*) depends not only on $\mathscr{S}$ but also on the metric used and is not a flow invariant.)

Note that if we had only required $a_{n} \rightarrow 0$, then (*) would be equivalent to uniform expansiveness relative to $\mathscr{P}$. It is easy to see that suspensions of subshifts or expansive toral automorphisms satisfy (*) when the metric is suitably chosen. A deeper result of Rufus Bowen shows that Axiom A flows also have this property.

(3.11) Proposition. Every Axiom A flow has a metric with which it satisfies (*).

Proof. By 1.6 of [1] every Axiom A flow (with correctly chosen metric $d$ ) has the property that there are constants $c>0$ and $\lambda>0$ such that, for each $\delta>0$, there is $\eta>0$ so that, if $x, y \in X, s: \mathbb{R} \rightarrow \mathbb{R}$ is continuous with $s(0)=0$ and $d(x t, y s(t)) \leq \eta$ for $|t| \leq L$, then

$$
d(y, x v) \leq c \exp (-\lambda L) \quad \text { for some }|v| \leq \delta .
$$

Find $c$ and $\lambda$ and choose $\rho_{0}=\delta<\frac{1}{2} \alpha$ with corresponding constant $\eta$. Let $t_{0}$ be such that $x t_{0}=x^{\prime}$ and $t_{i}$ such that $x t_{i}=\phi^{i}\left(x^{\prime}\right)(i \geq 1$ or $i \leq-1)$. Now define $s$ piecewise linear such that $y s\left(t_{i}\right)=y_{i}^{\prime}$ and $s(0)=0$. Now choosing a suitably small $\eta^{\prime}$ (independent of $s)$ we have $d\left(\phi^{i} x^{\prime}, y_{i}^{\prime}\right)<\eta^{\prime}$ implies $d(x t, y s(t)) \leq \eta$. If the first inequality holds for all $|i| \leq n$, the second holds for all $|t| \leq n \beta$ and thus

$$
d(x t, y t v) \leq c \exp (-\lambda(n \beta-2 \alpha)) \text { for }|t|<\alpha+2 \rho_{0} \text { and }|v|<\rho_{0} .
$$

So we define the sequence $\left\{a_{n}\right\}$ by

$$
a_{n}=c \exp (\lambda(2 \alpha)) \cdot \exp (-\lambda \beta n) .
$$

(3.12) TheOREM. Let $(X, \mathbb{R})$ have property $(*)$ and no periodic points. Then $(X, \mathbb{R})$ has no spiral orbits and thus it is a suspension of a subshift if it is minimal.

Proof. Suppose that $y=x T \in W_{\eta}^{s}(x)$. The idea of the proof is to show that, for large enough $N$, there is a neighbourhood of $\phi^{N}(x)$ contained in $D_{\rho}$ such that the positive semi-orbit of $\phi^{N}(x)$ keeps returning to this neighbourhood within bounded time. By looking at where these orbits cut the section containing $\phi^{N}(x)$ and choosing a convergent subsequence, we obtain a periodic point. 
Choose $\gamma>0$ such that, if $x \in T$ and $y \in B_{\gamma}(x)$, then $y \in D_{\rho}$ and $d\left(P_{\rho}(y), x\right)<\varepsilon$. Now choose $\rho_{0}<\rho$ in the definition of $(*)$ in such a way that the corresponding constant $\delta(=\eta$ of the definition) satisfies $a \in S$ and $b \in X$ and $d(a, b)<\delta$ then $d(a t, b t)<\frac{1}{2} \gamma$, where $|t|<\alpha+\rho$. Let $\delta^{\prime}$ be such that, if $a, b \in S$ and $d(a, b)<\delta^{\prime}$, then $d(a t, b t)<\delta$ for $|t|<2 \alpha$. Choose $M$ large enough so that $\sum_{i=M}^{\infty} a_{i}<\frac{1}{2} \gamma$. Choose $N>2 M$ large enough so that $y_{n}^{x} t \in D_{\rho}$ and $d\left(\left(\phi^{n} x\right) t, P_{\rho}\left(y_{n} t\right)\right)<\delta^{\prime}$ for all $n \geq \frac{1}{2} N,\left(\phi^{n} x\right) t \in S^{+}$ and $|t|<\alpha$. Moreover, choose $N$ large enough so that if $L$ is such that $\left(\phi^{N} x\right) L=y_{N}$, then $L>\alpha \beta /(\beta-\rho)$. (We may do this as we can assume that $\bar{t}_{\mathrm{n}} \rightarrow \infty$ where $\left(\phi^{n} x\right) \bar{t}_{n}=$ $y_{n}$.) Set $w=\phi^{N} x$ and $w_{0}=y_{N}$. We shall inductively define a sequence $\left\{w_{j}\right\}$ of points in the section containing $w$ such that $w_{j+1}=w_{j} t_{j}$ for some $\beta<t_{j}<L(1+\rho / \beta)$ and $d\left(w_{j}, w_{j+1}\right) \rightarrow 0$. This clearly implies the existence of a periodic point.

Define $z_{1}^{1}=y_{N+1}, z_{2}^{1}=y_{N+2}, \ldots, z_{k}^{1}=y_{N+k}$, where $\left(\phi^{k} w\right)_{\chi}=w_{0}, 0<\chi<\alpha$ and $k$ is chosen to be maximal with respect to this property. Now we can find $\eta_{0}$ with $\left|\eta_{0}\right| \leq \rho_{0}$ such that $d\left(w, w_{0} \eta_{0}\right) \leq a_{M}$ because $d\left(\phi^{i} w,\left(w_{0}\right)_{i}^{w}\right) \leq \delta$ for all $i \geq 0$ and also for $-M<i<0$ as $N-M \geq \frac{1}{2} N$. Similarly, for $j=1, \ldots, k$, we can find $\eta_{i}^{1}$ with $\left|\eta_{i}^{1}\right| \leq \rho_{0}$ and $d\left(\phi^{j} w, z_{i}^{1} \eta_{j}^{1}\right)<a_{M}$. Now

$$
d\left(w, z_{k}^{1}\left(\chi+\eta_{0}\right)\right) \leq d\left(w, w_{0} \eta_{0}\right)+d\left(\left(\phi^{k} w\right)\left(\chi+\eta_{0}\right), z_{k}^{1}\left(\chi+\eta_{0}\right)\right) \leq a_{M}+\frac{1}{2} \gamma<\gamma .
$$

Thus $z_{k}^{1}\left(\chi+\eta_{0}\right) \in D_{\rho}$ and define $w_{1}=P_{\rho}\left(z_{k}^{1}\left(\chi+\eta_{0}\right)\right)$.

Now suppose that $w_{0}, w_{1}, \ldots, w_{n-1}$ in $S$ have been constructed, and $z_{1}^{1}, \ldots, z_{k}^{1}, z_{1}^{2}, \ldots, z_{k}^{2}, \ldots, z_{1}^{n}, \ldots, z_{l}^{n}$ in $S^{+}\left(z_{i}^{i}\right.$ belongs to the same section for $j>0$ and a fixed $i$ with $1 \leq i \leq k$ ) have been constructed in such a way that

$$
\begin{gathered}
d\left(z_{i}^{i} \eta_{i}^{i}, z_{i}^{j+1} \eta_{i}^{j+1}\right) \leq a_{j+M}\left(1 \leq j \leq n-1,1 \leq i \leq k,\left|\eta_{i}^{j}\right| \leq \rho\right), \\
d\left(\phi^{i} w, z_{i}^{m} \eta_{i}^{m}\right)<\gamma \quad \text { for } 1 \leq i \leq k \text { and each } m>0
\end{gathered}
$$

and if $\left(\phi^{i} w\right) t_{i}=\phi^{i+1} w$ and $z_{i}^{j} s_{i}^{i}=z_{i+1}^{j}$ then $\left|s_{i}^{j}-t_{i}\right|<\rho$. We construct $z_{l+1}^{n}\left(w_{n+1}\right.$ if $l=k$ ). Define

$$
z=z_{l}^{n}\left(s_{l}^{n-1}+\eta_{l+1}^{n-1}\right)
$$

Clearly

$$
d\left(z, z_{l+1}^{n-1} \eta_{l+1}^{n-1}\right)<\delta
$$

since $d\left(z_{l}^{n-1}, z_{l}^{n}\right)<\delta^{\prime}$. By the condition on $L$, each time we complete a new $z$ cycle to $k$ terms we increase the number of returns of $w$ to $T^{+}$by at least one. Thus the last inequality holds for all forward returns of $z_{l}^{n-1}$ to $T^{+}$and for at least $M+n-1$ backward returns. Thus, by the definition of $(*)$,

$$
d\left(z \eta, z_{l+1}^{n-1} \eta_{l+1}^{n-1}\right)=d\left(z_{l}^{n}\left(s_{l}^{n-1}+\eta_{l+1}^{n-1}+\eta\right), \quad z_{l}^{n-1}\left(s_{l}^{n-1}+\eta_{l+1}^{n-1}\right)\right) \leq a_{M+n-1}
$$

for some $|\eta| \leq \rho_{0}$. Now

$$
\begin{aligned}
d\left(\phi^{l+1} w, z \eta\right) & \leq d\left(\phi^{l+1} w, z_{l+1}^{1} \eta_{l+1}^{1}\right)+d\left(z_{l+1}^{1} \eta_{l+1}^{1}, z_{l+1}^{2} \eta_{l+1}^{2}\right)+\cdots+d\left(z_{l+1}^{n-1} \eta_{l+1}^{n-1}, z \eta\right) \\
& \leq a_{M}+a_{M+1}+\cdots+a_{M+n-1}<\frac{1}{2} \gamma
\end{aligned}
$$

so $z \eta \in D_{\rho}$. Set $z_{l+1}^{n}=P_{\rho}(z \eta), \eta_{l+1}^{n}$ such that $\left|\eta_{l+1}^{n}\right|<\rho$ and $z_{l+1}^{n} \eta_{l+1}^{n}=z \eta$, and $s_{l}^{n}$ such that $z_{l}^{n} s_{l}^{n}=z_{l+1}^{n}$. It remains only to check that $\left|s_{l}^{n}-t_{l}\right|<\rho$. Since $d\left(\phi^{l} w, z_{l}^{n}\right)<\varepsilon$, $z_{l}^{n}\left(t_{l}+\eta^{\prime}\right)$ belongs to the same section as $\phi^{l+1} w$ for some $\eta^{\prime}$ with $\left|\eta^{\prime}\right|<\rho . z_{l}^{n} s_{l}^{n}=z_{l+1}^{n}$ 
belongs to this section. Now

$$
z_{l}^{n}\left(t_{l}+\eta^{\prime}\right)\left(s_{l}^{n-1}+\eta_{l+1}^{n-1}+\eta-\eta_{l+1}^{n}-t_{l}-\eta^{\prime}\right)=z_{l+1}^{n}=z_{l}^{n} s_{l}^{n} .
$$

Now

$$
\left|s_{l}^{n-1}+\eta_{l+1}^{n-1}+\eta-\eta_{l+1}^{n}-t_{l}-\eta^{\prime}\right| \leq 5 \rho<\zeta
$$

so

$$
z_{l}^{n}\left(t_{l}+\eta^{\prime}-s_{l}^{n}\right)=z_{l}^{n} \quad \text { or } \quad\left|t_{l}-s_{l}^{n}\right|=\left|\eta^{\prime}\right|<\rho
$$

as required. Thus the inductive construction is complete and the result follows.

\section{Real-expansive flows and finite topological dimension}

In this section we use our section approach in conjunction with the properties of the whole space $X$, to show that, if $X$ supports a real-expansive flow, it must have finite topological dimension. This gives an exact parallel to Mañé's result for homeomorphisms. We continue to use the notation of $\S 2$.

(4.1) LEMMA. Let $(X, \mathbb{R})$ be real expansive and let $\varepsilon_{2}>0$ be less than some expansive constant. Let $0<\varepsilon_{1}<\varepsilon_{2}$. Then there is $\delta>0$ such that, if $x \in T, y \in S, d(x, y) \leq \delta$ and for some $n>0$ we have

$$
\varepsilon_{1} \leq \max \left\{d\left(\phi^{j} x, y_{j}\right) ; 0 \leq j \leq n\right\} \leq \varepsilon_{2},
$$

then $d\left(\phi^{n} x, y_{n}\right) \geq \delta$.

Proof. If not, we can find sequences $\left\{x^{n}\right\} \subset T$ and $\left\{y^{n}\right\} \subset S$ and sequences of integers $\left\{m_{n}\right\}$ and $\left\{l_{n}\right\}$ with $m_{n}>l_{n}>0$ such that

$$
\begin{gathered}
d\left(x^{n}, y^{n}\right) \rightarrow 0, d\left(\phi^{m_{n}} x^{n}, y_{m_{n}}^{n}\right) \rightarrow 0, \\
d\left(\phi^{l_{n}} x^{n}, y_{l_{n}}^{n}\right) \geq \varepsilon_{1} \quad \text { and } d\left(\phi^{m} x^{n}, y_{m}^{n}\right) \leq \varepsilon_{2} \quad \text { for } 0 \leq m \leq m_{n} .
\end{gathered}
$$

Note that flow continuity ensures that $l_{n} \rightarrow \infty$ and also that $m_{n}-l_{n} \rightarrow \infty$ as $n \rightarrow \infty$. Choosing a subsequence if necessary we can assume $\phi^{l_{n}} x^{n} \rightarrow x, y_{l_{n}}^{n} \rightarrow y$ and for a given integer $k, \phi^{l_{n}+k} x^{n} \rightarrow \phi^{s_{k}} x$. By lemma $2.9 y_{l_{n}+k}^{n} \rightarrow y_{s_{k}}^{x}$.

Thus, ensuring that $l_{n}$ and $m_{n}-l_{n}$ are sufficiently large,

$$
d\left(\phi^{s_{k}} x, y_{s_{k}}^{x}\right) \leq d\left(\phi^{s_{k}} x, \phi^{l_{n}+k} x_{n}\right)+d\left(\phi^{l_{n}+k} x^{n}, y_{l_{n}+k}^{n}\right)+d\left(y_{l_{n}+k}^{n}, y_{s_{k}}^{x}\right)
$$

and letting $n \rightarrow \infty$ we obtain

$$
d\left(\phi^{s_{k}} x, y_{s_{k}}^{x}\right) \leq \varepsilon_{2} .
$$

As this holds for any $k$ and $x \neq y$ we have a contradiction.

(4.2) TheOrem. If $(X, \mathbb{R})$ is a real-expansive flow then $X$ has finite topological dimension.

Proof. Let $\varepsilon_{2}$ be less than some expansive constant and let $\varepsilon_{1}=\frac{1}{2} \varepsilon_{2}$. Choose $\delta>0$ as in lemma 4.1 and choose $\delta^{\prime}>0$ such that if $x, y \in S$ and $d(x, y) \geq \delta$ then $d(x, y t) \geq \delta^{\prime}$ for all $|t| \leq 2 \alpha$. Also let $0<\eta<\varepsilon$ be such that $x, y \in S$ and $d(x, y)<\eta$ implies $d(x t, y t)<\delta^{\prime}$ for any $|t| \leq \alpha$.

Now let $\left\{U_{i}, i=1, \ldots, l\right\}$ be an open cover of $X$ such that $\delta\left(U_{i}\right)<\eta$, and $U_{i} \subset$ $T\left[-\frac{1}{2} \alpha,+\frac{1}{2} \alpha\right]$ for some $T \in \mathscr{T}$ for each $1 \leq i \leq l$. (We can assume this last condition can be satisfied because we can slightly modify lemma 2.4 so that $X=\bigcup_{i=1}^{n} T_{i}^{*}(0, \alpha)$.) 
Define

$$
U_{i j}^{n}=\left(U_{i}\right) n \alpha \cap\left(U_{j}\right)(-n \alpha) \cap T,
$$

where $T$ is a fixed member of $\mathscr{T}$. Thus, for each $n,\left\{U_{i j}^{n} ; 1 \leq i \leq l, 1 \leq j \leq l\right\}$ is an open cover of $T$.

We now define a sequence $\left\{\delta_{n}\right\}$ such that:

(i) if $x \in T, y \in S$ and $d(x, y)<\delta_{n}$, then $y_{i}$ is defined and $d\left(\phi^{i} x, y_{i}\right) \leq \varepsilon_{1}$ for all $|i| \leq(1+n) \alpha / \beta$

(ii) if $x, y \in S$ and $d(x, y)<\delta_{n}$, then if for some $z \in T, d\left(\phi^{i} z, x_{i}^{z}\right)<\varepsilon_{1}$ for all $|i| \leq(1+n) \alpha / \beta$, then $y_{i}^{z}$ is defined for all $|i| \leq(1+n) \alpha / \beta$ and $d\left(x_{i}^{z}, y_{i}^{z}\right)<\varepsilon_{1}$. If $x_{i}^{z}=x s_{i}$ and $y_{i}^{z}=y \bar{s}_{i}$ then $\left|s_{i}-\bar{s}_{i}\right|<\frac{1}{2} \alpha$ for all $|i| \leq(1+n) \alpha / \beta$.

Now form the $\delta_{n}$ components of each $U_{i j}^{n}$, i.e. $x \sim y$ if there is a sequence $x=x_{0}, x_{1}, \ldots, x_{p}=y$ such that $d\left(x_{i}, x_{i+1}\right) \leq \delta_{n}$ for each $0 \leq i \leq p-1$ and each $x_{i} \in$ $U_{i j}^{n}$. Taking $U_{i j}^{n k}$ as the equivalence, we observe that we obtain a new open cover of $T$. Now if $x \in T$, then $x$ belongs to at most $l^{2}$ sets of this new cover (for $n$ fixed) since, for fixed $i$ and $j, x$ can belong to at most one $U_{i j}^{n k}$.

We complete the proof that $X$ has topological dimension of at most $l^{2}-1$ by proving $\lim _{n \rightarrow \infty}\left(\sup _{i, j, k} \delta\left(U_{i j}^{n k}\right)\right)=0$.

If not, we can find $\xi>0$ and values of $n>N(\xi)(N(\xi)$ is the integer $N(\delta)$ corresponding to $\delta=\xi$ and $\eta=\varepsilon_{1}$ in corollary 2.11) such that, for some $i, j, k, \delta\left(U_{i j}^{n k}\right)>\xi$. Choose such values for $n, i, j$ and $k$ and points $x, y \in U_{i j}^{n k}$ with $d(x, y)>\xi$. Let $x=x^{0}, x^{1}, \ldots, x^{p}=y$ be a $\delta_{n}$ chain from $x$ to $y$ in $U_{i j}^{n k}$. Now $x(n \alpha) \in U_{i} \subset T\left[-\frac{1}{2} \alpha,+\frac{1}{2} \alpha\right]$ so $x(n \alpha+\gamma) \in T$ for some $|\gamma| \leq \frac{1}{2} \alpha$ and let $J(x)$ be the number of returns of $x$ to $T^{+}$in time $n \alpha+\gamma$. Now for each $1 \leq r \leq p$ such that $x_{i}^{r}$ is defined (relative to $x$ ) for all $|i| \leq J(x)$, let

Note that

$$
A_{r}=\max \left\{d\left(\phi^{i} x, x_{i}^{r}\right) ;|i| \leq J(x)\right\} \text {. }
$$

$$
(1+n) \alpha / \beta \geq J(x) \geq n-1 \geq N(\xi) .
$$

Now $A_{1}$ is defined and $A_{1} \leq \varepsilon_{1}$ by the choice of $\delta_{n}$. Suppose $A_{r} \leq \varepsilon_{1}$ for some $1 \leq r \leq p-1$. Then $x_{i}^{r+1}$ is defined for each $|i| \leq J(x)$ and so $A_{r+1}$ is defined. Let $r$ be such that $A_{r^{\prime}} \leq \varepsilon_{1}\left(r^{\prime}<r\right)$ and $A_{r}>\varepsilon_{1}$. Such an $r$ exists as otherwise $A_{p}$ is well defined and $A_{p} \leq \varepsilon_{1}$, which contradicts $d\left(x, x^{p}\right)>\xi$ and the choice of $N(\xi)$. Next note that $\left|A_{r}-A_{r-1}\right| \leq \varepsilon_{1}$. For otherwise $A_{r}>\varepsilon_{1}+A_{r-1}$ and say $A_{r}=d\left(\phi^{i} x, x_{i}^{r}\right)$ and $A_{r-1}=d\left(\phi^{j} x, x_{j}^{r-1}\right)$, then

so that

$$
d\left(\phi^{i} x, x_{i}^{r}\right)>\varepsilon_{1}+d\left(\phi^{i} x, x_{i}^{r-1}\right)
$$

$$
\begin{aligned}
d\left(\phi^{i} x, x_{i}^{r-1}\right) & \geq d\left(\phi^{i} x, x_{i}^{r}\right)-d\left(x_{i}^{r}, x_{i}^{r-1}\right)>\varepsilon_{1}+d\left(\phi^{i} x, x_{j}^{r-1}\right)-d\left(x_{i}^{r}, x_{i}^{r-1}\right) \\
& \geq d\left(\phi^{i} x, x_{j}^{r-1}\right)
\end{aligned}
$$

and this contradicts the choice of $j$ for $A_{r-1}$. Now $A_{r} \leq 2 \varepsilon_{1}=\varepsilon_{2}$ and so by lemma 4.1 we have

$$
d\left(\phi^{J(x)} x, x_{J(x)}^{r}\right) \geq \delta .
$$

Now by the choice of $\delta_{n}, x_{i}^{j}$ is well defined for $j=1,2, \ldots, r$ and $i=0, \ldots, J(x)$. Let $s_{j}$ be such that $x^{j} s_{j}=x_{J(x)}^{i}$. Note that $\left|s_{0}-n \alpha\right| \leq \frac{1}{2} \alpha$. Suppose that $m$ is the first 
positive integer less than or equal to $r$ such that $\left|s_{m}-n \alpha\right|>\frac{1}{2} \alpha$. Again by the choice of $\delta_{n},\left|s_{m-1}-s_{m}\right| \leq \frac{1}{2} \alpha$ and thus

$$
\left|s_{m}-n \alpha\right| \leq\left|s_{m-1}-n \alpha\right|+\left|s_{m-1}-s_{m}\right| \leq \alpha .
$$

But now $x^{m} n \alpha \in U^{j}$ so $y=x^{m}(n \alpha+\chi) \in T \subset S$ for some $\chi$ with $|\chi| \leq \frac{1}{2} \alpha$, and $y(-n \alpha-\chi+s) \in S$. But

$$
\left|-n \alpha-\chi+s_{m}\right| \leq 2 \alpha<\zeta
$$

so that

$$
\left|s_{m}-n \alpha\right|=|\chi| \leq \frac{1}{2} \alpha .
$$

This contradiction shows that

$$
\left|s_{r}-n \alpha\right| \leq \frac{1}{2} \alpha .
$$

Thus if we let $s=n \alpha-s_{r}$ then $|s| \leq \alpha$ and $x_{J(x)}^{r} s \in U_{j}$ and $x^{r}(n \alpha+\gamma)=x_{J(x)}^{r}(s+\gamma)$. Now

$$
d\left(x_{J(x)}^{r}(s+\gamma), \phi^{J(x)}(x)\right)=d\left(x^{r}(n \alpha+\gamma), x(n \alpha+\gamma)\right) \geq \delta^{\prime}
$$

since $|s+\gamma| \leq 2 \alpha$ and so

$$
d\left(x^{r} n \alpha, x n \alpha\right) \geq \eta,
$$

but $x^{r} n \alpha$ and $x n \alpha$ are both in $U_{j}$ which is a contradiction.

Research of the first author was supported by NSF grant no. MCS78-00403 and of the second author by a post-doctoral bursary from the C.S.I.R.

\section{REFERENCES}

[1] R. Bowen. Periodic orbits for hyperbolic flows. Amer. J. Math. 94 (1972), 1-30.

[2] R. Bowen. Symbolic dynamics for hyperbolic flows. Amer. J. Math. 95 (2) (1973), 429-460.

[3] R. Bowen \& P. Walters. Expansive one-parameter flows. J. of Diff. Equations 12 (1972), 180-193.

[4] H. B. Keynes \& M. Sears. Modelling expansion in real flows. Pacific J. of Math. 85 (1979), 111-124.

[5] R. Mañé. Expansive homeomorphisms and topological dimension. Trans. AMS 252 (1979), 313319.

[6] H. Whitney. Regular families of curves. Ann. of Math. 34 (1933), 244-270. 